\title{
Erratum: Complex Langevin dynamics and zeroes of the fermion determinant
}

\author{
Gert Aarts, ${ }^{a}$ Erhard Seiler, ${ }^{b}$ Dénes Sexty ${ }^{c}$ and Ion-Olimpiu Stamatescu ${ }^{d, e}$ \\ ${ }^{a}$ Department of Physics, College of Science, Swansea University, \\ Singleton Park, Swansea, SA2 8PP, U.K. \\ ${ }^{b}$ Max-Planck-Institut für Physik (Werner-Heisenberg-Institut), \\ Föhringer Ring 6, D-80805 München, Germany \\ ${ }^{c}$ Bergische Universität Wuppertal, \\ Gaußtraße 20, D-42119 Wuppertal, Germany \\ ${ }^{d}$ Institut für Theoretische Physik, Universität Heidelberg, \\ Philosophenweg 16, D-69120 Heidelberg, Germany \\ e FEST, \\ Schmeilweg 5, D-69118 Heidelberg, Germany \\ E-mail: g.aarts@swan.ac.uk, ehs@mppmu.mpg.de, sexty@uni-wuppertal.de, \\ i.o.stamatescu@thphys.uni-heidelberg.de
}

ERRATUM TO: JHEP05(2017)044

ARXIV EPRINT: 1701.02322

L.L. Salcedo (private communication) has pointed out that eq. (3.18) of the paper must be incorrect and hence the discussion surrounding those two equations must be faulty. We agree with this judgement; the right hand side of eq. (3.18) is in fact 0 , by the argument leading to eq. (3.10).

In order to understand the failure of complex Langevin in this case, one has to consider not the equilibrium distribution, but quantity eq. (2.10), referring to finite Langevin time. This is where a boundary term arises, due to the fact that the evolved observable will have an essential singularity. The relevant discussion is given earlier in the paper after eq. (2.24).

We note that the error does affect neither the rest of the paper nor the general conclusion.

Open Access. This article is distributed under the terms of the Creative Commons Attribution License (CC-BY 4.0), which permits any use, distribution and reproduction in any medium, provided the original author(s) and source are credited. 